\title{
SALÁRIOS E RISCO DE ACIDENTES DE TRABALHO: EVIDÊNCIAS DE DIFERENCIAIS COMPENSATÓRIOS PARA A INDÚSTRIA MANUFATUREIRA*
}

\author{
Luiz Alberto Esteves ${ }^{\S}$
}

\begin{abstract}
RESUMO
O objetivo deste trabalho é fornecer evidências empíricas para a hipótese de diferenciais compensatórios de salários para a indústria brasileira de transformação. O argumento básico é o de que indústrias que expõem seus trabalhadores a maiores riscos de acidentes de trabalho deveriam compensá-los com maiores salários. Os resultados obtidos neste trabalho corroboram os fatos estilizados da literatura, ou seja, a ausência do controle da heterogeneidade não observada dos trabalhadores subestima fortemente os valores dos prêmios compensatórios e, após tal controle, a hipótese de diferenciais compensatórios é amplamente corroborada.
\end{abstract}

Palavras-chave: diferenciais compensatórios, diferencial de salários interindustriais, determinação de salários.

\begin{abstract}
The aim of this paper is to provide empirical evidences of compensating wage differentials for Brazilian manufacturing firms. This theory states that higher injury risk industries would pay higher wages in order to attract workers - it implies a tradeoff between wages and job amenities. The empirical evidences of this paper are similar to stylized facts of the literature: omitted variables bias provides underestimated values for the injury risks coefficient, but after controlling the unobserved heterogeneity of workers the coefficient becomes positive and significant.
\end{abstract}

Keywords: compensating wage differentials, industry wage differentials, wage determination.

JEL classification: J31.

* O autor é grato aos comentários e sugestões dos pareceristas anônimos. Os erros e omissões são de minha exclusiva responsabilidade.

$\S$ Universidade Federal do Paraná e Università di Siena. Endereço para contato: Avenida Celso Garcia, 5885, bloco 2, ap. 51. CEP: 03063-000 - Tatuapé - São Paulo - SP. Endereço eletrônico: esteves@ufpr.br.

Recebido em fevereiro de 2007. Aceito para publicação em junho de 2008. 


\section{INTRODUÇÃO}

A idéia de que diferenças salariais entre trabalhadores surgiriam por conta da heterogeneidade das indústrias em fornecer ambientes seguros de trabalho foi desenvolvida originalmente por Adam Smith (1776), em $A$ riqueza das nações. O argumento básico é que indústrias que expõem seus trabalhadores a maiores riscos de acidentes de trabalho, ou no desenvolvimento de alguma doença de trabalho, deveriam compensá-los com maiores salários.

A literatura internacional fornece uma vasta relação de estudos empíricos neste sentido. Já no caso brasileiro, há uma considerável quantidade de trabalhos empíricos que analisam as várias formas e origens de diferenciais salariais entre trabalhadores, ${ }^{1}$ porém não é do nosso conhecimento qualquer trabalho que tenha fornecido evidências de diferenciais salariais compensatórios para o caso brasileiro.

Uma análise empírica adequada da relação entre riscos e salários requer a disponibilidade de dados que propiciem ao pesquisador contornar, minimamente, os possíveis vieses que surgem das estimativas econométricas utilizando mínimos quadrados ordinários (MQO). Será discutido, ao longo do trabalho, que a endogeneidade do risco, a omissão de variáveis e erros de medida podem enviesar fortemente os parâmetros obtidos com estimativas MQO.

A forma mais adequada de mensurar risco é controversa e depende da disponibilidade de dados. Na realidade, o risco engloba várias dimensões e a utilização de uma única medida pode não capturar a todas satisfatoriamente. Neste sentido, este trabalho utiliza duas medidas distintas de risco: (1) o porcentual de acidentes de trabalho para cada indústria (CNAE 2 dígitos), e (2) o porcentual de trabalhadores mortos ou aposentados em decorrência de acidentes ou doenças de trabalho para cada indústria.

A primeira medida tenta capturar a dimensão de risco associada à probabilidade de ocorrência do evento, ou seja, a probabilidade de um trabalhador sofrer uma acidente em determinada indústria. A segunda medida tentar capturar dimensão de risco associada à extensão do evento, ou seja, a gravidade ou irreversibilidade do dano.

O objetivo deste trabalho é fornecer evidências empíricas sobre a hipótese de diferenciais compensatórios de salários para a indústria brasileira. Como mencionado nos parágrafos anteriores, tal análise requer a utilização de métodos econométricos alternativos com o objetivo de contornar os tipos de vieses já mencionados. Neste sentido, este trabalho apresenta resultados obtidos a partir de diferentes métodos econométricos, a saber: (1) análise de corte seccional com mínimos quadrados ordinários - MQO, e (2) análise de dados de painel - efeitos fixos para trabalhadores.

Um objetivo secundário deste trabalho é mostrar que os dados da indústria brasileira corroboram um resultado bem conhecido da literatura sobre o tema: as estimativas de corte seccional com MQO subestimam fortemente os prêmios de risco das equações de salários.

Este artigo é divido em cinco seções, incluindo esta introdução. A segunda seção é dedicada à apresentação do referencial teórico. A terceira seção é dedicada à apresentação dos dados, bem como

1 Coelho e Corseuil (2002) fornecem um survey da literatura empírica nacional sobre as diversas origens de diferenciais de salários. 
das estatísticas descritivas das variáveis a serem utilizadas no trabalho. A quarta seção apresenta os resultados obtidos a partir das estimativas econométricas. A quinta e última seção é dedicada às conclusões e considerações finais.

\section{TEORIA}

Modelos de diferenciais compensatórios de salários são fornecidos por Rosen (1986) e Viscusi e Aldy (2003). ${ }^{2}$ Os argumentos por trás destes modelos podem ser sinteticamente apresentados nos termos apresentados por Ehrenberg (1985).

Consideremos um mundo em que o mercado de trabalho é competitivo, os trabalhadores detêm completa informação sobre os riscos de acidentes associados a cada emprego e não há barreiras de mobilidade entre estes.

Suponha também que as firmas diferem em termos tecnológicos e cada uma destas tecnologias expõe os trabalhadores a diferentes riscos de acidentes. Tais riscos podem ser reduzidos por meio de maiores despesas de prevenção e o custo marginal de redução de riscos varia entre as firmas.

Assuma que os trabalhadores valorem positivamente seus ganhos salariais esperados por período e negativamente a probabilidade de incorrerem em acidentes de trabalho. Assim, os trabalhadores migrariam para empresas que fornecessem uma combinação de ganhos e riscos que maximizassem seu bem-estar.

Caso o conjunto dos trabalhadores apresentasse as mesmas preferências, as empresas com maior risco de acidente deveriam pagar maiores salários para atrair trabalhadores. Deste modo, a mobilidade dos trabalhadores conduziria os mercados a praticarem diferenciais salariais compensatórios.

A idéia acima pode ser formalizada de uma maneira bem simples: suponhamos que a utilidade do trabalhador possa ser expressa pela seguinte função de utilidade:

$$
u=u(w, d), u_{w}>0 \text { e } u_{d}<0
$$

onde $u$ é a utilidade do trabalhador, $w$ é o salário e $d$ é o custo de um acidente de trabalho. Os sinais das derivadas parciais demonstram a utilidade do salário e a desutilidade de um acidente de trabalho.

Suponhamos, por simplicidade, que a forma funcional da utilidade do trabalhador seja:

$$
u=w-d
$$

Suponhamos que haja dois tipos de empresas: (i) aquelas empresas cuja tecnologia implica uma probabilidade zero de acidentes a seus trabalhadores, e (ii) aquelas empresas cuja tecnologia implica uma probabilidade positiva de acidentes de trabalho.

2 Uma discussão teórica sobre o tema é fornecida por Fernandes (2002). 
Dada esta heterogeneidade tecnológica das empresas, a utilidade do trabalhador empregado em uma empresa com risco/probabilidade zero de acidentes será:

$$
u^{s}=w^{s}
$$

onde o sobrescrito $s$ significa um ambiente seguro de trabalho.

Já a utilidade do trabalhador em uma empresa com probabilidade positiva de riscos de acidentes será:

$$
u^{i}=(1-\theta) w^{i}+\theta\left(w^{i}-d\right)
$$

onde o sobrescrito $i$ significa um ambiente insalubre de trabalho e $\theta$ é a probabilidade de o trabalhador incorrer em algum acidente de trabalho, onde $0<\theta \leq 1$.

Igualando as equações (4) e (5), podemos obter o diferencial de salário necessário para compensar a probabilidade de riscos de acidentes e tornar indiferente ao trabalhador a escolha de emprego em ambas as empresas:

$$
w^{i}-w^{s}=\theta d
$$

Verifica-se, na equação (5), que o diferencial de salários necessário para compensar os riscos de acidentes cresce à medida que a probabilidade de acidentes, $\theta$, e o custo de acidentes, $d$, são maiores.

\section{DADOS}

Os dados utilizados para os testes empíricos deste trabalho foram obtidos em duas diferentes fontes de informações. A primeira fonte de informações é o Relatório Anual de Informações Sociais (RAIS), para os anos de 1997, 1998 e 1999. Esta base dispõe de informações individuais de salário, gênero, idade e escolaridade para 499.062 observações de trabalhadores "full time blue collars" durante todo o período analisado. Estas observações referem-se a uma amostra de 244.218 trabalhadores diferentes, sendo que apenas 91.920 trabalhadores estão presentes em todos os anos da amostra.

A RAIS também disponibiliza informações dos estabelecimentos empregadores. Os trabalhadores de nossa amostra estão filiados a 615 empresas da indústria brasileira de transformação. Nossa amostra dispõe de informações sobre a localização e o código de atividade (CNAE 3 dígitos) destas empresas.

A segunda fonte de informações utilizada neste trabalho é proveniente dos Anuários Estatísticos de Acidentes de Trabalho do Ministério da Previdência Social, anos de 1997, 1998 e 1999. Estas estatísticas são fornecidas por CNAE 2 dígitos, o que possibilita um link com as informações da RAIS. 
As medidas de risco utilizadas neste trabalho são obtidas, para cada ano da amostra, da seguinte maneira: a primeira medida é um porcentual obtido a partir da razão entre os acidentes de trabalho por CNAE (2 dígitos) e o total de trabalhadores formais ${ }^{3}$ empregados em cada uma destas indústrias (os valores censitários de trabalhadores formais por CNAE foram obtidos no site institucional do Ministério do Trabalho e Emprego - Programa de Disseminação de Estatísticas do Trabalho, PDET); a segunda medida é obtida de forma análoga à primeira, porém utilizando informações de óbitos e aposentadorias decorrentes de acidentes ou doenças de trabalho.

\section{Figura 1 - Correlação \% de acidentes versus \% de mortes e aposentadorias}

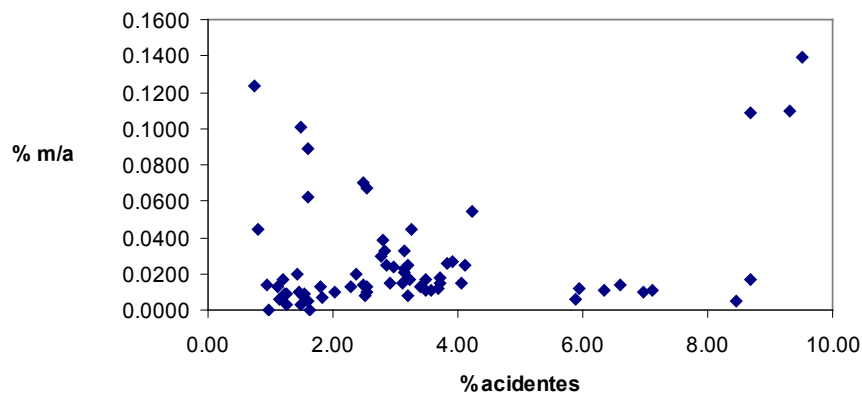

Cabe aqui fazer um comentário sobre as diferentes formas de obtenção da variável risco de acidentes na literatura. Alguns autores como Thaler e Rosen (1975), Brown (1980), Leigh (1981) e Arnould e Nichols (1983) utilizam, a exemplo do presente trabalho, informações censitárias de acidentes de trabalho. Já Hamermesh (1978), Viscusi (1979, 1980) e Fairris (1989) utilizam informações reportadas pelos próprios trabalhadores sobre o risco de acidentes de suas ocupações e encontram uma forte correlação entre salários e riscos. A literatura sugere que os resultados da correlação entre riscos e salários sejam sensíveis conforme a medida de risco utilizada. ${ }^{4}$

As estatísticas descritivas das variáveis a serem utilizadas neste trabalho são reportadas, por ano, na Tabela 1. Nas Tabela 2 e 3, são reportados os porcentuais de acidentes e porcentuais de mortes e aposentadorias por CNAE e ano, respectivamente.

3 Optou-se por considerar trabalhadores formais, pois as estatísticas de acidentes de trabalho são provenientes das comunicações de acidentes de trabalho (CAT). Tais comunicações são em sua grande maioria reportadas para trabalhadores formais com "carteira assinada".

4 Consultar Linnerooth (1979), Smith (1979), Brown (1980), Blomquist (1981), Rosen (1986) e Hwang, Reed e Hubbard (1992) para uma ampla discussão sobre medidas de risco, modelos de risco, diferentes métodos econométricos de estimação de prêmios, fatos estilizados, etc. 
Tabela 1 - Estatísticas descritivas

\begin{tabular}{lccc}
\hline Variáveis & 1997 & 1998 & 1999 \\
\hline Ln (salário-horário) & 1,22 & 1,29 & 1,19 \\
& $(0,67)$ & $(0,70)$ & $(0,70)$ \\
Escolaridade (em anos) & 6,59 & 6,39 & 6,33 \\
Gênero (\% homens) & $(3,07)$ & $(3,16)$ & $(3,29)$ \\
& 0,77 & 0,78 & 0,80 \\
Idade (em anos) & $(0,41)$ & $(0,41)$ & $(0,40)$ \\
Porcentual Acidentes/Emprego(CNAE 2 dígitos) & 33,03 & 34,34 & 34,51 \\
& $(11,15)$ & $(11,73)$ & $(11,65)$ \\
Porcentual de Mortes e Aposentadorias(CNAE 2 dígitos) & 3,26 & 3,26 & 3,00 \\
Observações & $(1,98)$ & $(2,05)$ & $(1,90)$ \\
\hline
\end{tabular}

Notas: (1) Desvio padrão entre parênteses abaixo das médias; (2) Salários a preços de 1999 ajustados pelos IPCA; (3) Pesos amostrais utilizados.

Pode-se verificar que as indústrias com maior incidência de acidentes (Tabela 2) são as de madeira, máquinas e equipamentos e fabricação de veículos automotores. Já as indústrias de vestuários e fabricação de máquinas e equipamentos de escritório e informática figuram entre os setores com menores incidências de acidentes. O ano de 1999 apresenta uma considerável redução dos acidentes de trabalho em quase todas as indústrias do setor de transformação.

\section{Tabela 2 - Porcentual de acidentes por indústria}

\begin{tabular}{llll}
\hline Indústria & 1997 & 1998 & 1999 \\
\hline Alimentos e Bebidas & 3,19 & 2,78 & 2,82 \\
Fumos & 3,10 & 2,56 & 1,62 \\
Têxteis & 2,54 & 2,29 & 2,48 \\
Vestuário & 1,24 & 1,11 & 0,94 \\
Couro e Calçados & 1,61 & 1,54 & 1,51 \\
Madeira & 9,52 & 9,31 & 8,70 \\
Papel e Celulose & 3,72 & 3,91 & 3,68 \\
Edição e Impressão & 1,60 & 1,48 & 1,26 \\
Químicos & 2,54 & 2,52 & 2,03 \\
Borracha e Plástico & 3,50 & 3,56 & 3,22 \\
Minerais Não-Metálicos & 3,27 & 3,13 & 2,80 \\
Metalurgia Básica & 2,87 & 2,98 & 2,37 \\
Produtos de Metal & 3,73 & 4,07 & 3,49 \\
Máquinas e Equipamentos & 7,12 & 6,96 & 5,93 \\
Máquinas Escritório e Informática & 1,14 & 0,99 & 0,80 \\
Aparelhos Elétricos & 3,40 & 3,21 & 2,90 \\
Material Eletrônico Comunicação & 1,79 & 1,83 & 1,46 \\
Médico-Hospitalares e Outros & 1,61 & 1,48 & 1,26 \\
Veículos Automotores & 6,61 & 6,35 & 5,90 \\
Equipamentos de Transporte & 1,42 & 1,19 & 0,75 \\
Móveis & 4,10 & 3,84 & 3,13 \\
\hline
\end{tabular}


$\mathrm{Na}$ Tabela 3, verifica-se que a indústria de madeira também apresenta o maior porcentual de óbitos e aposentadorias decorrentes de acidentes e doenças do trabalho, porém não há uma forte correlação entre os porcentuais de acidentes e os porcentuais de mortes e aposentadorias para o restante dos setores industriais (ver Figura 1). Outro ponto a ser destacado da Tabela 3 é que não há uma tendência generalizada de queda dos porcentuais no tempo, diferentemente do porcentual de acidentes vistos na Tabela 2.

Tabela 3 - Porcentual de mortes e aposentadorias por indústria

\begin{tabular}{lccc}
\hline Indústria & 1997 & 1998 & 1999 \\
\hline Alimentos e Bebidas & 0,0250 & 0,0301 & 0,0324 \\
Fumos & 0,0144 & 0,0102 & 0,0000 \\
Têxteis & 0,0670 & 0,0132 & 0,0140 \\
Vestuário & 0,0090 & 0,0130 & 0,0141 \\
Couro e Calçados & 0,0892 & 0,0084 & 0,0082 \\
Madeira & 0,1397 & 0,1099 & 0,1088 \\
Papel e Celulose & 0,0144 & 0,0265 & 0,0122 \\
Edição e Impressão & 0,0619 & 0,1005 & 0,0085 \\
Químicos & 0,0131 & 0,0082 & 0,0103 \\
Borracha e Plástico & 0,0113 & 0,0111 & 0,0171 \\
Minerais Não-Metálicos & 0,0444 & 0,0207 & 0,0388 \\
Metalurgia Básica & 0,0243 & 0,0238 & 0,0197 \\
Produtos de Metal & 0,0182 & 0,0150 & 0,0173 \\
Máquinas e Equipamentos & 0,0108 & 0,0099 & 0,0119 \\
Máquinas Escritório e Informática & 0,0061 & 0,0000 & 0,0440 \\
Aparelhos Elétricos & 0,0124 & 0,0077 & 0,0150 \\
Material Eletrônico Comunicação & 0,0124 & 0,0071 & 0,0103 \\
Médico-Hospitalares e Outros & 0,0054 & 0,0029 & 0,0032 \\
Veículos Automotores & 0,0134 & 0,0104 & 0,0061 \\
Equipamentos de Transporte & 0,0194 & 0,0168 & 0,1235 \\
Móveis & 0,0244 & 0,0255 & 0,0223 \\
\hline
\end{tabular}

\section{ANÁlise EMPÍricA}

\subsection{Análise de corte seccional}

Comecemos esta seção pela obtenção de estimativas da relação entre salários e risco de acidentes para cada ano da amostra. Para tal finalidade, utilizar-se-á uma equação de rendimentos com a seguinte especificação:

$$
\ln w_{i}=\alpha^{\prime} \mathbf{x}_{i}+\beta r_{i}^{j}+\varepsilon_{i}
$$


onde $\ln w_{\mathrm{i}}$ é o logaritmo do salário horário do trabalhador $i, \mathbf{x}_{i}$ é um vetor de variáveis relacionadas aos atributos do trabalhador $i, r_{i}^{j}$ é a medida de risco $j$ para a indústria onde o indivíduo $i$ está filiado, as letras gregas correspondem a coeficientes a serem estimados e $\varepsilon_{\mathrm{i}}$ é o erro aleatório.

Tal especificação econométrica foi aplicada separadamente para cada ano da amostra e para cada medida de risco. A Tabela 4 apresenta os resultados das estimativas dos coeficientes usando a medida de porcentual de acidentes, enquanto a Tabela 5 reporta os coeficientes obtidos com a medida de porcentual de mortes e aposentadorias.

A hipótese de diferenciais compensatórios é corroborada caso os coeficientes $\beta$ apresentem sinais positivos e significativos. No caso da Tabela 4 (onde o parâmetro $\beta$ é calculado para a variável porcentual de acidentes), apenas o coeficiente $\beta$ para o ano de 1997 apresentou sinal positivo e significativo, 0,004. As estimativas de $\beta$ para os demais períodos - 1998 e 1999 - apresentaram sinais negativos e significativos: $-0,005$ e $-0,015$, respectivamente.

Tabela 4 - Retornos sobre exposição a acidentes de trabalho - análise de corte seccional - variável dependente $=\ln$ (salário-horário)

\begin{tabular}{lccc}
\hline Variáveis & Regressão MQO 1997 & Regressão MQO 1998 & Regressão MQO 1999 \\
\hline Escolaridade (anos) & 0,05 & 0,05 & 0,06 \\
& $(0,0004)^{* * *}$ & $(0,0004)^{* * *}$ & $(0,0004)^{* * *}$ \\
Idade (anos) & 0,07 & 0,06 & 0,06 \\
Gênero & $(0,0006)^{* * *}$ & $(0,0006)^{* * *}$ & $(0,0006)^{* * *}$ \\
Porcentual de Acidentes & 0,43 & 0,45 & 0,40 \\
Dummies Localização & $(0,0029)^{* * *}$ & $(0,0033)^{* * *}$ & $(0,0035)^{* * *}$ \\
R $^{2}$ & 0,004 & $-0,005$ & $-0,015$ \\
F & $(0,0006)^{* * *}$ & $(0,0007)^{* * *}$ & $(0,0008)^{* * *}$ \\
Observações & Sim & Sim & Sim \\
\hline
\end{tabular}

Notas: (1) Erros padrão entre parênteses abaixo dos coeficientes; (2) Salários a preços de 1995 ajustados pelos IPCA; (3) Outros controles omitidos na tabela: (Idade) ${ }^{2}$; (4) Todas as regressões incluem uma constante; (5) Pesos amostrais utilizados; (6) Níveis de significância: $10 \%(*), 5 \%(* *)$ e $1 \%(* *)$.

No caso da Tabela 5 (onde o parâmetro $\beta$ é calculado para a variável porcentual de mortes e aposentadorias), nenhum dos coeficientes $\beta$ apresentou sinal positivo e significativo. As estimativas de $\beta$ para os todos os períodos - 1997, 1998 e 1999 - apresentaram sinais negativos e significativos: $-2,06,-0,724$ e $-1,296$, respectivamente.

Os resultados obtidos a partir das regressões MQO não fornecem evidências que corroborem a hipótese de diferenciais compensatórios de salários para a indústria brasileira de transformação. Ao contrário disto, tais resultados sugerem que trabalhadores filiados a indústrias com maior exposição ao risco de acidentes recebem salários inferiores aos seus pares filiados a indústrias menos insalubres. 
Tabela 5 - Retornos sobre exposição a acidentes de trabalho - análise de corte seccional - v-ariável dependente $=\ln ($ salário-horário)

\begin{tabular}{lccc}
\hline Variáveis & Regressão MQO 1997 & Regressão MQO 1998 & Regressão MQO 1999 \\
\hline Escolaridade (anos) & 0,05 & 0,05 & 0,06 \\
Idade (anos) & $(0,0004)^{* * *}$ & $(0,0004)^{* * *}$ & $(0,0004)^{* * *}$ \\
Gênero & 0,06 & 0,06 & 0,06 \\
Porcentual de Mortes e Aposentadorias & $(0,0006)^{* * *}$ & $(0,0006)^{* * *}$ & $(0,0006)^{* * *}$ \\
Dummies Localização & 0,44 & 0,45 & 0,40 \\
R & $(0,0028)^{* * *}$ & $(0,0032)^{* * *}$ & $(0,0034)^{* * *}$ \\
F & $-2,06$ & $-0,724$ & $-1,296$ \\
Observações & $(0,0424)^{* * *}$ & $(0,0436)^{* * *}$ & $(0,0550)^{* * *}$ \\
& Sim & Sim & Sim \\
\hline
\end{tabular}

Notas: (1) Erros padrão entre parênteses abaixo dos coeficientes; (2) Salários a preços de 1995 ajustados pelos IPCA; (3) Outros controles omitidos na tabela: (Idade) ${ }^{2}$; (4) Todas as regressões incluem uma constante; (5) Pesos amostrais utilizados; (6) Níveis de significância: $10 \%(*), 5 \%(* *)$ e $1 \%(* * *)$.

\subsection{Análise longitudinal}

Foi visto na seção anterior que a maioria dos testes com estimativas em MQO não corroboraram a hipótese de diferenciais compensatórios para o caso da indústria brasileira. Na realidade, a ocorrência de tais resultados a partir de estimativas em MQO é bem conhecida na literatura empírica sobre o tema. As estimativas em MQO tendem a subestimar os coeficientes da variável risco por dois motivos: (1) a endogeneidade da variável risco, e (2) o problema de omissão de variáveis. Um terceiro problema relacionado à subestimação dos prêmios refere-se ao erro de medida da variável risco.

O primeiro problema a ser considerado nos resultados apresentados na seção anterior é quanto à endogeneidade da variável risco de acidentes. Viscusi (1978) argumenta que há um efeito-riqueza na relação entre salários e riscos. Dado que a segurança seja um bem normal, espera-se que trabalhadores com maiores níveis de renda não provenientes do trabalho possam "adquirir" postos mais seguros. Tal riqueza também proporcionaria a estes trabalhadores o acesso a uma maior acumulação de capital humano, por exemplo, em termos de melhor qualidade da educação. Este efeitoriqueza pode, assim, estar enviesando os parâmetros obtidos por estimativas em MQO, devido à correlação dos resíduos com o risco de acidentes.

O problema da omissão de variáveis está associado ao problema de endogeneidade. Podemos imaginar que a produtividade do trabalhador seja decomposta em características observáveis e nãoobserváveis. Como ambas as características determinam os salários dos trabalhadores, aqueles mais produtivos se "auto-selecionam" em postos de trabalho com maior remuneração e melhor qualidade (menor exposição a riscos, por exemplo). O problema é que as estimativas de corte seccional em 
MQO ignoram o efeito que a produtividade decorrente de características não-observáveis exerce sobre os salários e seleção de postos de trabalho com melhor qualidade.

Os tipos de medidas utilizados neste trabalho podem também estar contribuindo para subestimar os parâmetros da variável risco na equação de salários. Infelizmente não dispomos de informações sobre risco de acidentes reportados pelos próprios trabalhadores para testar tal hipótese para o caso brasileiro.

Hwang, Reed e Hubbard (1992) demonstram analiticamente o viés decorrente de estimativas com MQO. Ao utilizar técnicas de simulação, estes autores apontam que tal viés pode subestimar os prêmios de risco em um fator de $50 \%$ ou mais, podendo até mesmo implicar a estimativa de coeficientes com sinais trocados - o que parece ser o caso deste trabalho.

Uma proposta de solução para o problema de omissão de variáveis é sugerida por Brown (1980), que tenta contornar o problema por meio do controle da heterogeneidade não-observável e constante no tempo dos trabalhadores em um painel de dados. Duncan e Holmlund (1983) seguem o mesmo procedimento. Na ausência de dados longitudinais, Garen (1988) sugere a utilização de variáveis instrumentais para contornar o problema da endogeneidade da variável risco.

A característica longitudinal dos dados disponíveis e a ausência de variáveis que atendam aos requisitos de exogeneidade dos instrumentais possibilitam que o presente trabalho tente contornar o problema de subestimação dos prêmios de risco mediante a utilização do controle da heterogeneidade não-observável e constante no tempo dos trabalhadores, conforme especificação do modelo abaixo:

$$
\ln w_{i t}=\alpha^{\prime} \mathbf{x}_{i t}+\beta r_{i t}^{j}+\eta_{i}+\lambda_{t}+\varepsilon_{i t}
$$

onde $\ln w_{\text {it }}$ é o logaritmo do salário-horário do trabalhador $i$ no período $t$, $\mathbf{x}_{i t}$ é um vetor de variáveis relacionadas aos atributos do trabalhador $i$ no período $t, r_{i t}^{j}$ é a medida de risco $j$ para a indústria onde o indivíduo $i$ está filiado no período $t, \eta_{\mathrm{i}}$ são os efeitos fixos dos trabalhadores, $\lambda_{t}$ são os efeitos fixos de tempo e $\varepsilon_{i t}$ é o erro aleatório.

A exemplo da especificação da equação 6, a hipótese de diferenciais compensatórios é corroborada caso os coeficientes $\beta$ apresentem sinais positivos e significativos.

A disponibilidade de uma identificação única para os trabalhadores formais brasileiros, o PIS (Programa de Integração Social), garante que seja possível acompanhar os trabalhadores da amostra ao longo do tempo, possibilitando assim o controle da heterogeneidade não-observável e constante no tempo.

Os resultados das estimativas com efeitos fixos de trabalhadores estão disponíveis na Tabela 6. A primeira coluna de resultados refere-se às estimativas com a medida de risco porcentual de acidentes, enquanto que a segunda coluna de resultados refere-se às estimativas com a medida de risco porcentual de mortes e aposentadorias. Em ambos os casos, os sinais dos coeficientes $\beta$ foram positivos e significativos -0,020 para o coeficiente da variável porcentual de acidentes e 0,307 para o coeficiente da variável porcentual de mortes e aposentadorias. 
Os valores dos coeficientes $\beta$, reportados na Tabela 6 , corroboram a hipótese de diferenciais compensatórios para o setor industrial brasileiro, bem como, ainda, um fato estilizado da literatura sobre o tema: as estimativas em MQO tendem a subestimar os prêmios de risco, podendo tal viés até mesmo implicar a troca dos sinais dos parâmetros estimados.

Tabela 6 - Retornos sobre exposição a acidentes de trabalho - análise longitudinal - variável dependente $=\ln$ (salário-horário)

\begin{tabular}{|c|c|c|}
\hline Variáveis & $\begin{array}{l}\text { Regressão Efeitos Fixos } \\
\text { Trabalhadores }\end{array}$ & $\begin{array}{l}\text { Regressão Efeitos Fixos } \\
\text { Trabalhadores }\end{array}$ \\
\hline Escolaridade (anos) & $\begin{array}{l}0,008 \\
(0,0005)^{* * *}\end{array}$ & $\begin{array}{l}0,008 \\
(0,0005)^{\star \star \star}\end{array}$ \\
\hline Idade (anos) & $\begin{array}{l}0,084 \\
(0,0016)^{\star * *}\end{array}$ & $\begin{array}{l}0,082 \\
(0,0016)^{\star \star *}\end{array}$ \\
\hline Porcentual de Acidentes & $\begin{array}{l}0,020 \\
(0,0024)^{\star \star *}\end{array}$ & \\
\hline Porcentual de Mortes/Aposentadorias & & $\begin{array}{l}0,307 \\
(0,0178)^{\star * *}\end{array}$ \\
\hline Dummies Localização & Sim & Sim \\
\hline Dummies de Ano & $\operatorname{Sim}$ & Sim \\
\hline Dummies de Indústria & Sim & Sim \\
\hline$R^{2}$ & 0,95 & 0,95 \\
\hline $\mathrm{F}$ & $629^{\star \star *}$ & $638^{* * *}$ \\
\hline Observações & 499062 & 499062 \\
\hline
\end{tabular}

Notas: (1) Erros padrão entre parênteses abaixo dos coeficientes; (2) Salários a preços de 1995 ajustados pelos IPCA; (3) Outros controles omitidos na tabela: (Idade) ${ }^{2}$; (4) Todas as regressões incluem uma constante; (5) Pesos amostrais utilizados; (6) Níveis de significância: $10 \%(*), 5 \%(* *)$ e $1 \%(* * *)$.

\section{CONCLUSÃO}

O objetivo deste trabalho foi fornecer evidências empíricas sobre a hipótese de diferenciais compensatórios de salários para a indústria brasileira. Embora tal assunto seja amplamente explorado na literatura internacional, poucas evidências empíricas são disponíveis para a economia brasileira e demais países em desenvolvimento.

Neste artigo, providencia-se uma série de resultados para a relação entre salários e risco, obtidos a partir de diferentes estimadores econométricos e diferentes medidas de risco.

A utilização de diferentes medidas de risco deve-se ao fato de o risco envolver várias dimensões e a utilização de uma única medida pode não capturar a todas satisfatoriamente. Neste sentido, este trabalho utilizou duas medidas distintas de risco: o porcentual de acidentes de trabalho (probabilidade do evento) e o porcentual de trabalhadores mortos ou aposentados em decorrência de acidentes ou doenças de trabalho (gravidade do evento). 
A utilização de diferentes métodos econométricos é justificada pelos fatos estilizados da literatura internacional, uma vez que é amplamente difundida a idéia de que estimativas de corte seccional em mínimos quadrados ordinários (MQO) resultam em parâmetros subestimados para o prêmio de risco.

Foram verificados ao longo deste trabalho os seguintes resultados: (1) estimativas de corte seccional em MQO geram parâmetros negativos e significativos, e (2) o controle da heterogeneidade não-observada e constante no tempo dos trabalhadores gera coeficientes positivos e significativos para os prêmios de risco. Estes resultados corroboram os fatos estilizados de que as estimativas em MQO tendem a subestimar os prêmios de risco, podendo tal viés até mesmo implicar a troca dos sinais dos parâmetros estimados.

Os resultados obtidos neste trabalho fornecem fortes evidências de que os trabalhadores brasileiros expostos a maiores riscos de acidentes ou danos graves recebem prêmios salariais com o objetivo de compensar suas posições em postos de trabalho insalubres.

\section{REFERÊNCIAS}

ARNOULD, R. J.; NICHOLS, L. M. Wage risk premiums and workers compensation: a refinement of estimates of compensating wage differential. Journal of Political Economy, v. 91, n. 2, p. 332-340, 1983.

BLOMQUIST, Glenn C. The Value of Human Life: An Empirical Perspective. Economic Inquiry, v. 19: 157-164, 1981.

BROWN, C. Equalizing differences in the labor market. Quarterly Journal of Economics, v. 941, p. 113134, 1980.

COELHO, A. M.; CORSEUIL, C.H. Diferenciais salariais no Brasil: um breve panorama. In: CORSEUIL, G. H. (Ed.). Estrutura salarial: aspectos conceituais e novos resultados para o Brasil. Rio de Janeiro: IPEA, 2002, p. 67-100.

DUNCAN, G. J.; HOLMLUND, B. Was Adam Smith right after all? Another test of the theory of compensating wage differentials. Journal of Labor Economics, v. 1, 4, p. 366-379, 1983.

EHRENBERG, R. Workers compensation, wages, and the risk of injury. In: NBER Working Papers n.1538, 1985.

FAIRRIS, D. Compensating wage differentials in the union and nonunion sectors. Industrial Relations, v. 28, n. 3, p. 356-372, 1989.

FERNANDES, R. Desigualdade salarial: aspectos teóricos. In: CORSEUIL, G. H., (Ed.). Estrutura salarial: aspectos conceituais e novos resultados para o Brasil. Rio de Janeiro: IPEA, 2002, p. 1-50.

GAREN, J. Compensating wage differentials and the endogeneity of job riskiness. Review of Economics and Statistics, v. 70, n. 1, p. 916, 1988.

HAMERMESH, D.S. Economic aspects of job satisfaction. In: ASHENFELTER, O.; OATES, W. (Ed.). Essays in labor market analysis. New York: John Wiley \& Sons, 1978, p. 53-62.

HWANG, H.; REED, W. R.; HUBBARD, C. Compensating wage differentials and unobserved productivity. Journal of Political Economy, v. 100, p. 835-858, 1992.

LEIGH, J. P. Compensating wages for occupational injuries and diseases. Social Science Quarterly, v. 62, n. 4, p. 772-778, 1981. 
LINNEROOTH, Joanne. The value of human life: a review of the models. Economic Inquiry, v. 17, p. 52-74, 1979.

ROSEN, S. The theory of equalizing differences. In: ASHENFELTER, O.; LAYARD, R. (Ed.). Handbook of labor economics. North Holland, 1986.

SMITH, Adam. A riqueza das nações: investigação sobre sua natureza e suas causas. São Paulo: Abril Cultural, 1776 - 1983. v. 1.

SMITH, Robert S. Compensating wage differentials and public policy: a review. Industrial and Labor Relations Review, v. 32, p. 339-352, 1979.

THALER, R.; ROSEN, S. The value of saving a life: evidence from labor market. In: TERLECKYJ, N. E. (Ed.). Household production and consumption., New York: Columbia University Press, 1975, p. 265-300.

VISCUSI, W. K. Wealth effects and earning premiums for job hazards. Review of Economics and Statistics, v. 603, p. 408-416, 1978.

. Employment hazards: an investigation of market performance. Cambridge, MA: Harvard University Press, 1979.

. Union, labor market structure, and the welfare implications of the quality of work. Journal of Labor Research, v. 1, n. 1, p. 175-192, 1980.

.; ALDY, J. E. The value of statistical life: a critical review of market estimates throughout the world. Journal of Risk and Uncertainty, v. 27, p. 5-76, 2003. 